\title{
Risk of venous thromboembolism and myocardial infarction associated with factor $V$ Leiden and prothrombin mutations and blood type
}

\author{
Birgitte F. Sode MD PhD, Kristine H. Allin MD PhD, Morten Dahl MD DMSc, Finn Gyntelberg MD DMSc, \\ Børge G. Nordestgaard MD DMSc
}

\section{- ABSTRACT \\ Background: $A B O$ blood type locus has been reported to be an important genetic determi- nant of venous and arterial thrombosis in genome-wide association studies. We tested the hypothesis that $A B O$ blood type alone and in combination with mutations in factor $V$ Lei- den R506Q and prothrombin G20210A is asso- ciated with the risk of venous thromboem- bolism and myocardial infarction in the general population.}

Methods: We used data from 2 Danish studies that followed members of the general public from 1977 through 2010. We obtained the genotype of 66001 white participants for ABO blood type, factor $V$ Leiden R506Q and prothrombin G20210A. We calculated hazard ratios (HRs) and population attributable risk. Our main outcome measures were venous thromboembolism and myocardial infarction.

Results: The multivariable adjusted HR for venous thromboembolism was 1.4 (95\% confidence interval [Cl] 1.3-1.5) for non-O blood type (v. O blood type). For the factor $\mathrm{V}$ Leiden R506Q mutation, the adjusted HR was 2.2
(95\% Cl 2.0-2.5) for heterozygous participants and $7.0(95 \% \mathrm{Cl} 4.8-10)$ for homozygous participants (v. participants without the mutation). For prothrombin G20210A, the adjusted HR was $1.5(95 \% \mathrm{Cl} 1.2-1.9)$ for heterozygous participants and $11(95 \% \mathrm{Cl} 2.8-44)$ for homozygous participants (v. participants without the mutation). When we combined $A B O$ blood type and factor $\mathrm{V}$ Leiden $\mathrm{R} 506 \mathrm{Q}$ or prothrombin G20210A genotype, there was a stepwise increase in the risk of venous thromboembolism (trend, $p<0.001$ ). The population attributable risk of venous thromboembolism was $20 \%$ for $A B O$ blood type, $10 \%$ for factor $V$ Leiden R506Q and 1\% for prothrombin G20210A. Multivariable adjusted HRs for myocardial infarction by genotypes did not differ from 1.0.

Interpretation: $\mathrm{ABO}$ blood type had an additive effect on the risk of venous thromboembolism when combined with factor $V$ Leiden R506Q and prothrombin G20210A mutations; blood type was the most important risk factor for venous thromboembolism in the general population.
Competing interests: None declared.

This article has been peer reviewed.

Correspondence to: Børge G. Nordestgaard, boerge.nordestgaard@ regionh.dk

CMAJ 2013. DOI:10.1503 /cmaj.121636
$\mathrm{G}$ enome-wide association studies have reported that ABO blood type locus is an important genetic determinant of venous and arterial thrombosis, ${ }^{1,2}$ leading to renewed interest in the association between ABO blood type and venous and arterial thrombosis. This challenges conventional thoughts on genetic screening for thrombophilia, which presently does not include ABO blood type.

Individuals with an A or B blood type have an increased risk of venous thromboembolism and myocardial infarction compared with individuals with O blood type ${ }^{3-6}$ Earlier studies concluded that $\mathrm{ABO}$ antigen expression determines von
Willebrand factor levels, ${ }^{7-11}$ however, recent findings from genome-wide association studies suggest that $\mathrm{ABO}$ antigens may also exert their effect through other pathways. ${ }^{12-16}$ Both factor $\mathrm{V}$ Leiden R506Q and prothrombin G20210A mutations have been consistently associated with increased risk of venous thrombosis but not consistently associated with the risk of arterial thrombosis. ${ }^{17-19}$

In this study, we tested the hypothesis that $\mathrm{ABO}$ blood type, alone and in combination with the factor V Leiden R506Q and prothrombin G20210A mutations, is associated with the risk of venous thromboembolism and myocardial infarction in the general population. 


\section{Methods}

\section{Study cohort}

The study was approved by Herlev Hospital, Copenhagen University Hospital and by Danish ethical committees, and the study was conducted according to the Declaration of Helsinki. Written informed consent was obtained from all participants.

All study participants were white and of Danish descent. The participants were randomly selected from 2 similar, but independent, study cohorts recruited from the adult Danish general population. The Copenhagen General Population Study $(n=56960)$ is a prospective study initiated in $2003,{ }^{20}$ and the Copenhagen City Heart Study $(n=9041)$ is a prospective study initiated in $1976 .{ }^{20,21}$ Participants aged 20 100 years were selected based on the Central Population Register number. All participants answered similar questionnaires, which were each reviewed with an examiner. Objective clinical parameters (current smoking status, body mass index [BMI], diabetes mellitus, hypertension, use of oral contraceptives) were measured by similar methods. Participants in the 2 studies were recruited from different parts of Copenhagen, and there was no overlap of individuals between the 2 studies. Follow-up was $100 \%$ complete in both studies.

\section{Outcome measures}

Our definition of venous thromboembolism was based on the International Classification of Diseases (ICD), 8th and 10th editions (ICD-8: 451.00, 451.08-09, 451.90, 451.92, 671.01-03, 671.08-09; ICD-10: I80.1-3, O22.3, O87.1). Myocardial infarction was defined as ICD-8 code 410 and ICD-10 codes I21-I22. In both studies, information on all hospital admission dates and diagnoses (including emergency department, outpatient and hospital admission) and all death dates and diagnoses were obtained from the Danish National Patient Register and the national Danish Register of Causes of Death using the unique Danish Central Person Register numbers from January 1, 1977, through August 8,2010 . These ICD codes have been previously studied in these cohorts, using identical criteria for case ascertainment. ${ }^{20,22}$

\section{Other covariables}

We obtained information on current smoking status, BMI, diabetes mellitus, hypertension and use of oral contraceptives. Diabetes mellitus was selfreported, and the use of oral hypoglycemic agents or insulin, a nonfasting plasma glucose greater than $11 \mathrm{mmol} / \mathrm{L}$ and/or a diagnosis of diabetes mellitus was collected from the national registers (ICD-8 codes 249-250; ICD-10 codes E10-E11, E13-E14). Hypertension was classified as systolic blood pressure above $140 \mathrm{~mm} \mathrm{Hg}$, diastolic blood pressure above $90 \mathrm{~mm} \mathrm{Hg}$, or the use of antihypertensive medication. We recorded the use of oral contraceptives among premenopausal women.

\section{Genotyping and biochemical analysis}

We determined $A B O$ genotype from singlenucleotide polymorphisms in the $\mathrm{ABO}$ gene using TaqMan assays (Applied Biosystems). Participants with type $\mathrm{O}$ blood were defined as being homozygous for polymorphism rs8176719. Those with type B blood were homozygous for rs8176746 or heterozygous for both rs8176719 and rs8176746. Participants with type A blood had neither polymorphism. Participants with $\mathrm{AB}$ blood type were heterozygous for rs8176746 only. The genotypes were validated by sequencing.

All genotypes were in Hardy-Weinberg equilibrium, and the frequencies of the $\mathrm{A}, \mathrm{B}, \mathrm{AB}$ and $\mathrm{O}$ blood types were consistent with those in the Danish general population. ${ }^{23}$ Genotyping for factor $\mathrm{V}$ Leiden R506Q (mutation rs6025) and prothrombin G20210A (mutation rs1799963) was done as reported previously ${ }^{24,25}$ or by using TaqMan assays (Applied Biosystems).

We calculated the plasma level of low-density lipoprotein cholesterol using the Friedewald equation if triglycerides were below $4 \mathrm{mmol} / \mathrm{L}$; they were measured directly at higher levels. We measured the following using standard hospital assays: plasma levels of total cholesterol, highdensity lipoprotein cholesterol, platelets, mean platelet volume, leukocytes, coagulation factors (II, VII, X), international normalized ratio, activated partial thromboplastin time, high-sensitivity C-reactive protein and complement C3. All analyses were performed on fresh samples on the day of attendance. Data on these variables were only available for participants in the Copenhagen General Population Study.

\section{Statistical analysis}

We assessed the association between the factor $\mathrm{V}$ Leiden R506Q and prothrombin G20210A mutations, $\mathrm{ABO}$ blood type, and risk of venous thromboembolism or myocardial infarction. We used log-rank tests and Cox regression models with age as the time scale, automatically adjusted for age. For the trend tests, participants without the mutations (noncarriers) were coded as 0 , heterozygous participants were coded as 1 and homozygous participants were coded as 2 . Cox models were left truncated with delayed entry in 
1977, and individuals were censored at events, death, emigration or end of follow-up (August 8, 2010). We excluded individuals who had an event before 1977. Multivariable models were adjusted for age, sex, current smoking, BMI, systolic blood pressure and diabetes mellitus. Missing values among potential confounders $(<1 \%)$ used in the multivariable models were imputed using multiple imputation. Tests of interaction in Cox multivariable models were performed by introducing a 2-factor interaction term.

We calculated population attributable risks based on significant hazard ratios (HRs) using the formula $[f(\mathrm{HR}-1)] /[1+f(\mathrm{HR}-1)]$, where $f$ is the frequency of the risk factor in the population at baseline, and HR is the HR for venous thromboembolism. For ranking the risk factors by population attributable risk, we used dichotomized baseline risk factors (current v. nonsmoker; hypertension v. no hypertension; diabetes v. no diabetes; BMI > 25 v. $\leq 25 \mathrm{~kg} / \mathrm{m}^{2}$; O blood type v. non-O blood type; heterozygous or homozygous for factor V Leiden R506Q mutation v. no mutation; heterozygous or homozygous for prothrombin G20210A mutation v. no mutation). Because age was adjusted for automatically in the
Cox regression model, we did not include this risk factor in the ranking analysis.

We evaluated differences in mean values for primary hemostasis, coagulation and inflammatory parameters using Wilcoxon rank sum tests and tests for trend.

\section{Results}

Individuals in the Copenhagen City Heart Study were more likely to be current smokers, have lower systolic blood pressure, higher plasma cholesterol levels and use statins less often than individuals in the Copenhagen General Population Study (Table 1).

\section{Venous thromboembolism}

In the combined studies, which included 2279 venous thromboembolism events, the multivariable adjusted HR for venous thromboembolism was 1.4 (95\% confidence interval [CI] 1.3-1.5) among individuals with non-O blood type (v. O blood type) ( $p<0.001$; Table 2$)$. Among individuals with the factor V Leiden R506Q mutation, the corresponding HRs (v. noncarriers) were 2.2 (95\% CI 2.0-2.5) for heterozygous individuals

Table 1: Characteristics of the included participants

\begin{tabular}{|c|c|c|c|}
\hline \multirow[b]{2}{*}{ Characteristic } & \multicolumn{3}{|c|}{ Median (IQR)* } \\
\hline & $\begin{array}{c}\text { Copenhagen General } \\
\text { Population Studyt } \\
n=56960\end{array}$ & $\begin{array}{c}\text { Copenhagen City } \\
\text { Heart Study } \\
n=9041\end{array}$ & Combined \\
\hline No. of women & 31537 & 5025 & 36562 \\
\hline No. of men & 25423 & 4016 & 29439 \\
\hline Age, yr & $60(50-70)$ & $60(47-70)$ & $60(50-70)$ \\
\hline Smoking status, $¥$ no. (\%) & $12001(21)$ & 4419 (49) & $16420(25)$ \\
\hline $\mathrm{BMI}$ & $26(23-29)$ & $25(23-28)$ & $26(23-28)$ \\
\hline $\mathrm{BMI}>25 \mathrm{~kg} / \mathrm{m}^{2}$, no. (\%) & $32245(57)$ & $4541(50)$ & $36786(56)$ \\
\hline Systolic blood pressure, $\mathrm{mm} \mathrm{Hg}$ & $140(128-152)$ & $136(122-152)$ & $140(126-152)$ \\
\hline Diabetes mellitus, no. (\%) & $2964(5)$ & $416(5)$ & $3380(5)$ \\
\hline Use of oral contraceptives, § no. (\%) & $1917(6)$ & $335(7)$ & $2252(6)$ \\
\hline Cholesterol, $\mathrm{mmol} / \mathrm{L}$ & $5.6(4.9-6.3)$ & $6.1(5.3-6.9)$ & $5.6(4.9-6.4)$ \\
\hline $\begin{array}{l}\text { Low-density lipoprotein cholesterol, } \\
\mathrm{mmol} / \mathrm{L}\end{array}$ & $3.2(2.6-3.8)$ & $3.7(3.0-4.5)$ & $3.2(2.6-3.9)$ \\
\hline $\begin{array}{l}\text { High-density lipoprotein cholesterol, } \\
\mathrm{mmol} / \mathrm{L}\end{array}$ & $1.6(1.3-1.9)$ & $1.5(1.2-1.9)$ & $1.6(1.3-1.9)$ \\
\hline Triglycerides, $\mathrm{mmol} / \mathrm{L}$ & $1.4(1.0-2.1)$ & $1.5(1.1-2.2)$ & $1.4(1.0-2.1)$ \\
\hline $\begin{array}{l}\text { Use of lipid lowering medication, no. } \\
(\%)\end{array}$ & $5593(10)$ & $286(3)$ & 5879 (9) \\
\hline \multicolumn{4}{|c|}{$\begin{array}{l}\text { Note: } \mathrm{BMI}=\text { body mass index, } \mathrm{IQR}=\text { interquartile range. } \\
\text { *Unless stated otherwise. } \\
\text { tValues were obtained at study examination in both the Copenhagen General Population Study and the Copenhagen City } \\
\text { Heart Study. } \\
\text { FIncludes past and current smokers. } \\
\text { §Among premenopausal women. }\end{array}$} \\
\hline
\end{tabular}


and 7.0 (95\% CI 4.8-10) for homozygous individuals (trend, $p<0.001$ ). Among those with the prothrombin G20210A mutation, the adjusted HR was 1.5 (95\% CI 1.2-1.9) for heterozygous individuals and 11 (95\% CI 2.8-44) for homozygous individuals (trend, $p<0.001$ ) (Table 2). Similar results were observed in the individual studies (Appendix 1, available at www.cmaj.ca /lookup/suppl/doi:10.1503/cmaj.121636/-/DC1) and when deep venous thrombosis and pulmonary embolism were considered separately (Appendix 2, available at www.cmaj.ca/lookup/suppl/doi :10.1503/cmaj.121636/-/DC1).

When we combined ABO blood type with factor V Leiden R506Q or prothrombin G20210A genotypes, there were stepwise increases in the risk of venous thromboembolism (trend, $p<$ 0.001) (Figure 1). When we combined factor $\mathrm{V}$ Leiden R506Q and prothrombin G20210A geno- types, there was also a stepwise increase in the risk of venous thromboembolism (trend, $p<0.001$ ). There was no statistically significant multiplicative interaction for any combination of genotypes.

\section{Myocardial infarction}

In the combined studies (2708 myocardial infarction events), the multivariable adjusted HR for myocardial infarction was 1.1 (95\% CI 1.0-1.1) among individuals with non-O blood type (v. O blood type) ( $p=0.2$; Table 2). Among those with the factor V Leiden R506Q mutation, the corresponding adjusted HRs (v. those without the mutation) were 1.0 (95\% CI 0.9-1.2) for heterozygous individuals and 0.9 (95\% CI 0.4-2.1) for homozygous individuals (trend, $p=1.0$ ). Among those with the prothrombin G20210A mutation, the HR was 1.3 (95\% CI 1.0-1.7) for heterozygous individuals $(p=0.04)$. There were

Table 2: Risk of venous thromboembolism and myocardial infarction among participants in the Copenhagen General Population Study and the Copenhagen City Heart Study

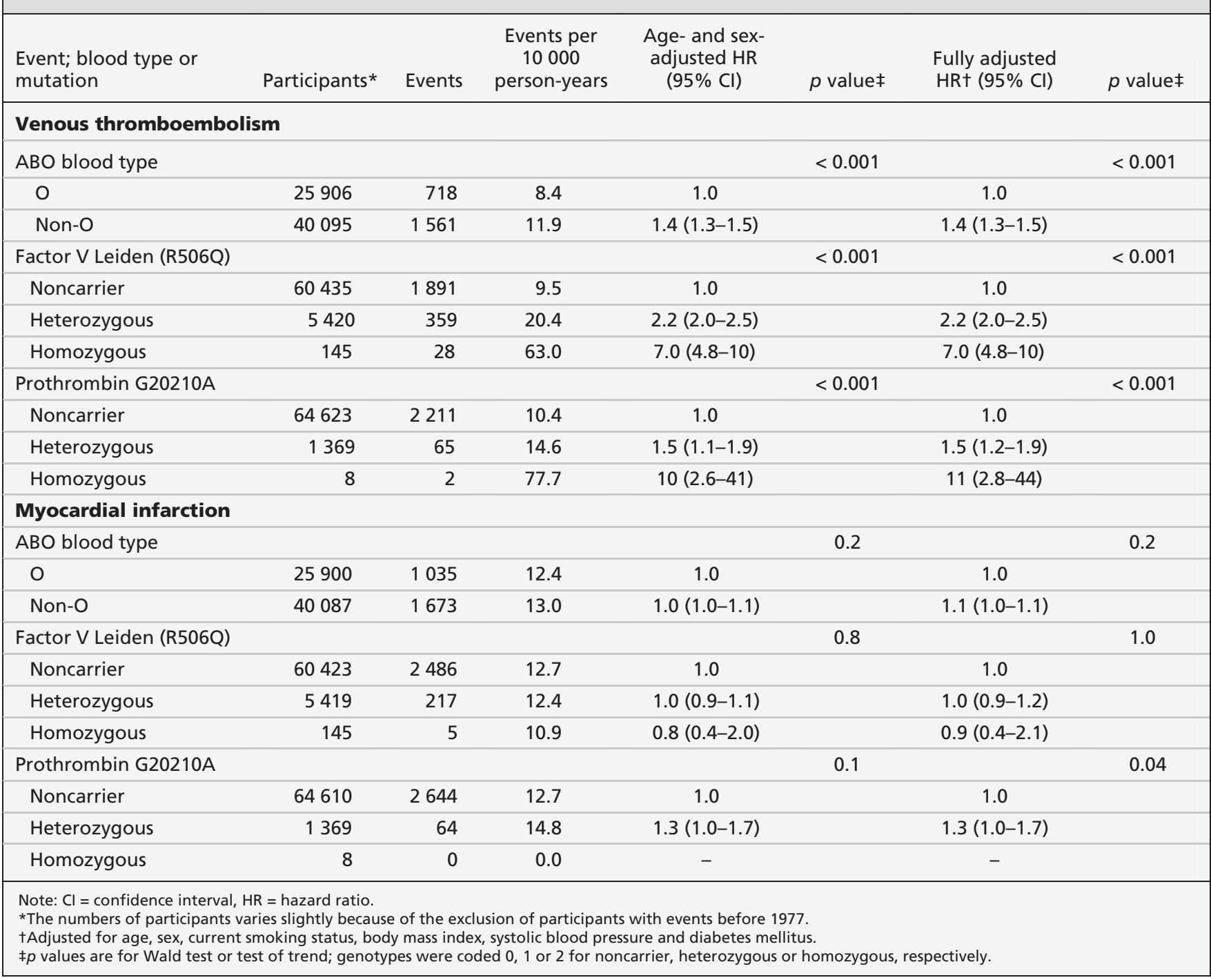


no myocardial infarction events among the 8 participants who were homozygous for the prothrombin G20210A mutation (Table 2). Similar results were observed in the individual studies (Appendix 3, available at www.cmaj.ca/lookup /suppl/doi:10.1503/cmaj.121636/-/DC1).

When we combined ABO blood type and factor V Leiden R506Q or prothrombin G20210A genotype, there was no consistent increased risk of myocardial infarction (Figure 2). This was also true for combinations of factor V Leiden R506Q and prothrombin G20210A genotypes. There was no statistically significant multiplicative interaction for any combination of genotypes.

Risk factors for venous thromboembolism We ranked the risk factors for venous thromboembolism according to population attributable risk in the combined studies (Figure 3). The pop- ulation attributable risk of venous thromboembolism was $20 \%$ for ABO blood type, $10 \%$ for factor V Leiden R506Q and 1\% for prothrombin G20210A. For comparison, BMI of $25 \mathrm{~kg} / \mathrm{m}^{2}$ or higher and current smoking had population attributable risks of $16 \%$ and $6 \%$, respectively.

\section{Primary hemostasis, coagulation and inflammatory parameters}

We measured biochemical markers of primary hemostasis, coagulation and inflammation according to ABO blood type, factor V Leiden R506Q and prothrombin G20210A in the Copenhagen General Population Study (Table 3). Although we observed some differences between genotypes in these parameters, the effect sizes were generally modest. Non-O versus O blood type was associated with reduced activated partial thromboplastin time.

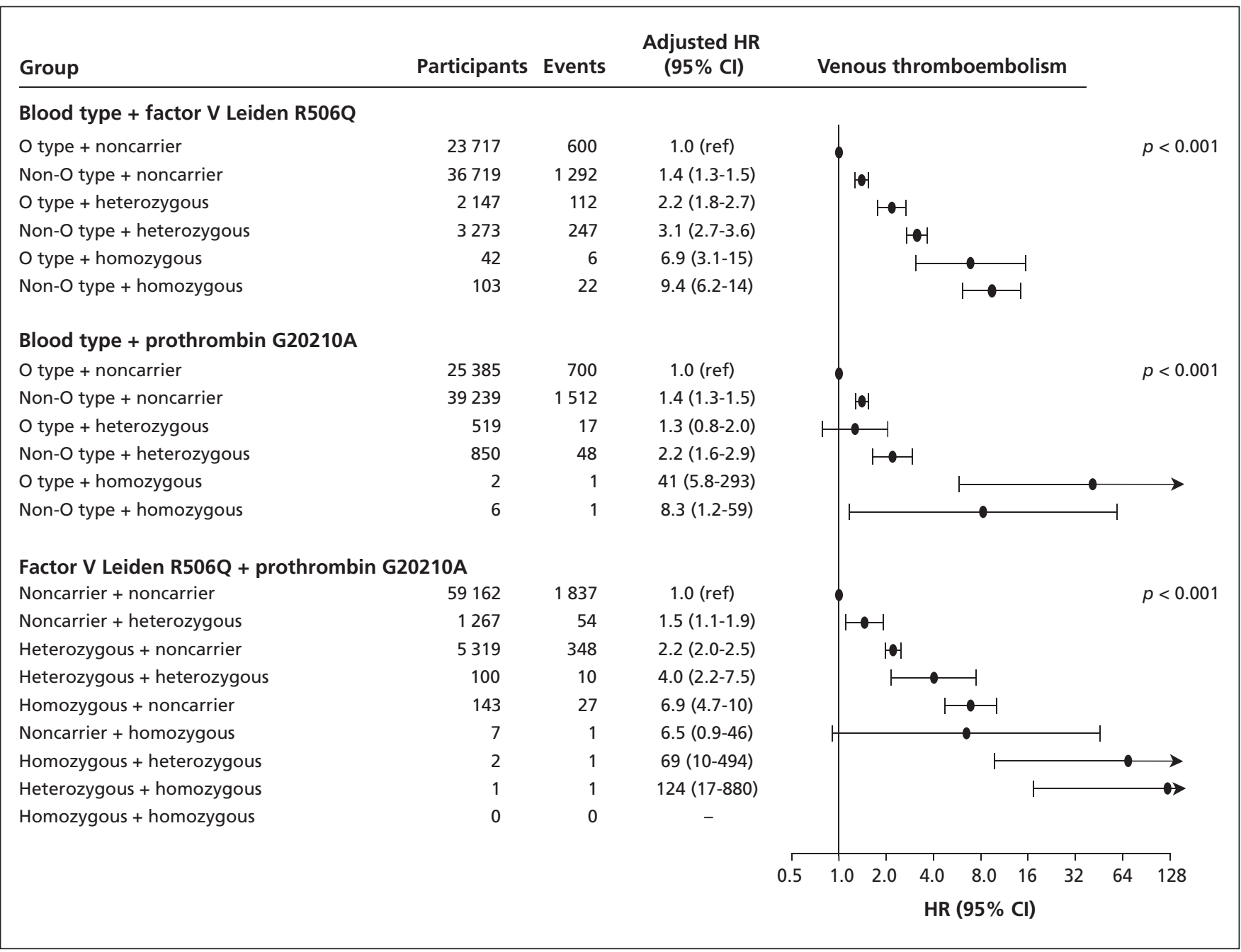

Figure 1: Risk of venous thromboembolism by ABO blood type, factor V Leiden R506Q and prothrombin G20210A mutations among 66001 individuals in the Copenhagen General Population Study and the Copenhagen City Heart Study. Hazard ratios (HR) with 95\% confidence intervals (Cls) were adjusted for age, sex, current smoking status, body mass index, systolic blood pressure and diabetes mellitus. $p$ values are for trend across genotype combinations coded 0 through 6 or 0 through 8 , depending on the number of genotype combinations. 


\section{Interpretation}

We found an additive effect of non-O blood type and prothrombin G20210A genotype on the risk of venous thromboembolism. We also found an 11 -fold increased risk of venous thromboembolism among participants who were homozygous for the prothrombin G20210A mutation; previous general population studies have been underpowered to address this question. The additive effect of $\mathrm{ABO}$ blood type and factor V Leiden R506Q mutation and the additive effect of factor V Leiden R506Q and prothrombin G20210A mutations on the risk of venous thromboembolism confirms the findings of previous studies. ${ }^{19,25,26}$

We also found that non-O blood type was the most important risk factor for venous thromboembolism, constituting $20 \%$ of the population attributable risk for venous thromboembolism, compared with $10 \%$ for factor V Leiden R506Q and $1 \%$ for prothrombin G20210A mutations. This suggests that $\mathrm{ABO}$ blood type should be included in genetic screening for thrombophilia.

In accordance with the results of a previous study, ${ }^{27}$ we found that the population attributable risks of venous thromboembolism for a BMI of 25 or higher was large. In our study, ABO blood type, factor V Leiden R506Q and prothrombin G20210A mutations were not consistently associated with myocardial infarction, which is also consistent with the findings of previous studies. ${ }^{17-19}$ A meta-analysis that included more than 60000 patients with coronary artery disease and more than 90000 controls found a significant, although slight association, between this disease and the presence of factor V Leiden and G20210 prothrombin mutations. ${ }^{19}$ There was, however, an indication of publication bias in these studies.

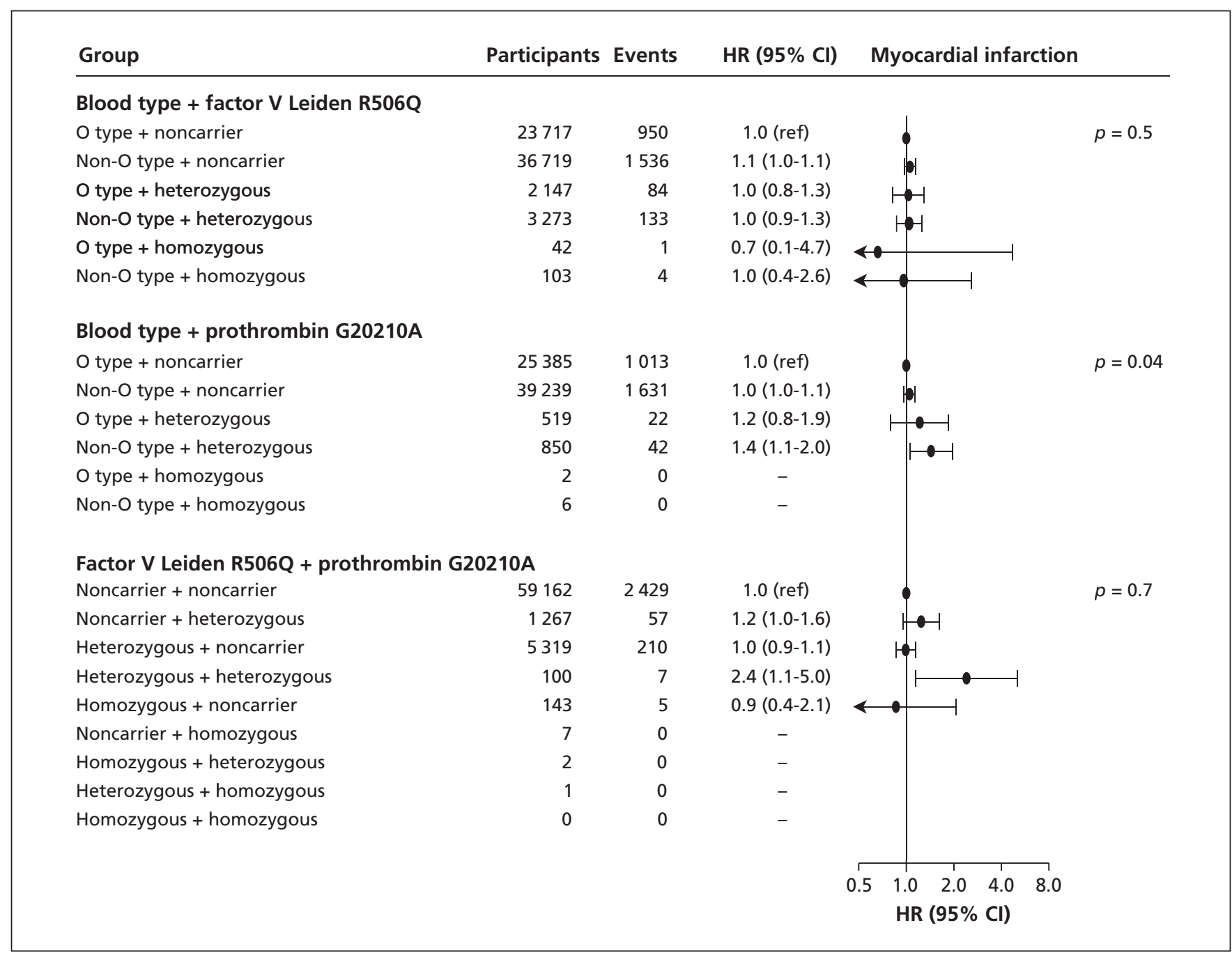

Figure 2: Risk of myocardial infarction by ABO blood type, factor V Leiden R506Q and prothrombin G20210A mutations among 66001 individuals in the Copenhagen General Population Study and the Copenhagen City Heart Study. Hazard ratios (HR) with 95\% confidence intervals (Cls) were adjusted for age, sex, current smoking status, body mass index, systolic blood pressure and diabetes mellitus. $p$ values are for trend across genotype combinations coded 0 through 6 or 0 through 8 , depending on the number of genotype combinations. 
Mechanistically, the association between $\mathrm{ABO}$ blood type and venous thromboembolism can be partly explained by increased levels of von Willebrand factor and/or factor VIII in the blood of non-O individuals, ${ }^{11}$ because higher levels of these factors are risk factors for venous thromboembolism..$^{28,29}$ Von Willebrand factor distributes $\mathrm{ABO}$ antigens on the surface of the membrane ${ }^{30}$ which may cause reduced clearance of von Willebrand factor by ADAMST13 metalloproteinase. As well, $66 \%$ of the total variation in plasma levels of von Willebrand factor is genetically determined, and $30 \%$ of this genetic component can be explained by ABO blood type. ${ }^{8}$ Individuals with a non-O blood type have $25 \%-30 \%$ higher plasma levels of von Willebrand factor than individuals with type O blood; ${ }^{8,911}$ this likely explains our observation of reduced activated partial thromboplastin time among individuals with non-O blood type compared to O blood type. However, although the increased risk of venous thromboembolism among those with non-O blood type has consistently been linked to the association with von Willebrand factor levels, ${ }^{31}$ recent findings from genome-wide association studies suggest that $\mathrm{ABO}$ antigens may also exert their effect through other pathways. ${ }^{12-16}$

Factor V Leiden R506Q is a single base mutation in the factor $\mathrm{V}$ gene, which eliminates 1 of its 3 activated protein $\mathrm{C}$ cleavage sites. ${ }^{17}$ Consequently, factor $\mathrm{V}$ is inactivated at a lower rate, leading to increased production of thrombin and increased risk of venous thromboembolism. Prothrombin G20210A is a single base mutation in the untranslated region of the gene promoter, which leads to increased production of prothrombin,,$^{32}$ causing prothrombin levels to be elevated by about $30 \%$ in heterozygous individuals and $70 \%$ in homozygous individuals. ${ }^{18}$ This increase ultimately leads to increased risk of venous thromboembolism.

\section{Strengths and limitations}

Our study included a large number of participants from genetically homogenous cohorts followed over a long period, which allowed us to calculate prospective risk estimates, even for rare mutations. Follow-up was 100\% complete for the included cohorts. As well, the results from the 2 included prospective cohorts were consistent, which supports that our findings are valid.

Our findings may not necessarily apply to ethnic backgrounds and nationalities other than white Danes. However, because we studied genetic factors that are found universally but at different frequencies, we expect that our results should be generalizable to most backgrounds and nationalities.

\begin{tabular}{|c|c|c|c|c|c|c|c|c|c|c|c|c|c|c|c|}
\hline 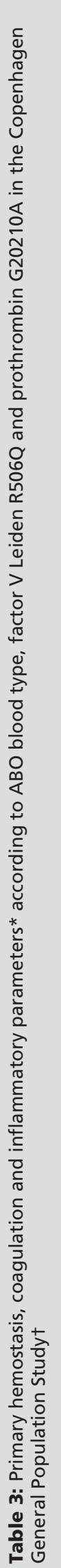 & 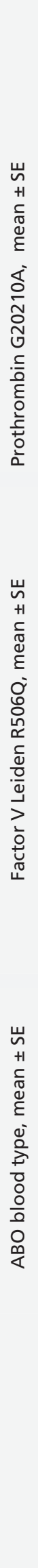 & 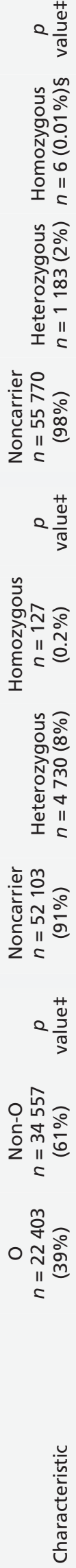 & 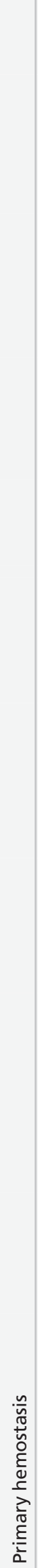 & $\begin{array}{l}\frac{1}{2} \\
0 \\
\frac{5}{0} \\
\frac{0}{2} \\
\frac{0}{0} \\
\frac{\pi}{2}\end{array}$ & 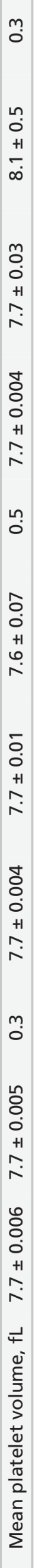 & 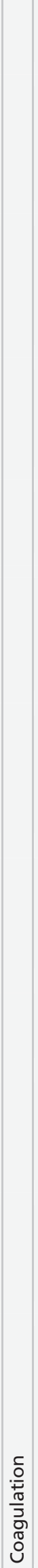 & 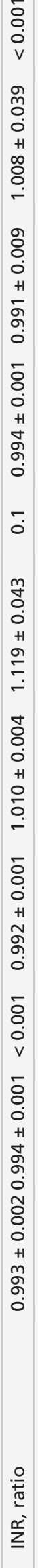 & 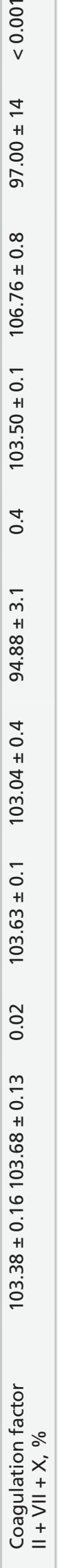 & $\begin{array}{l}m \\
0 \\
0 \\
+1 \\
+ \\
\dot{N}\end{array}$ & 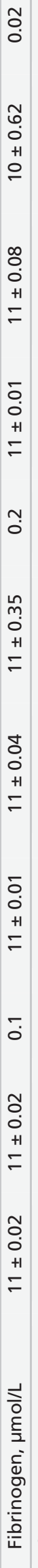 & 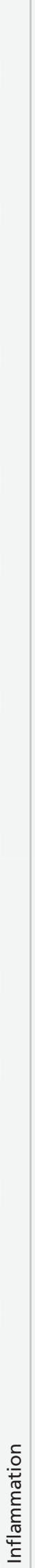 & $\begin{array}{l}m \\
0 \\
0 \\
+1 \\
\infty \\
i j \\
\\
m \\
0 \\
0 \\
+1 \\
\hat{j} \\
\hat{j}\end{array}$ & 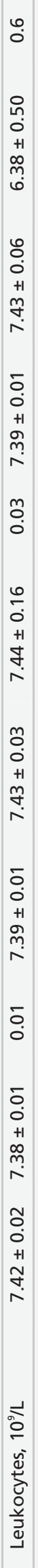 & 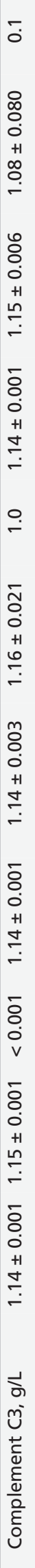 & 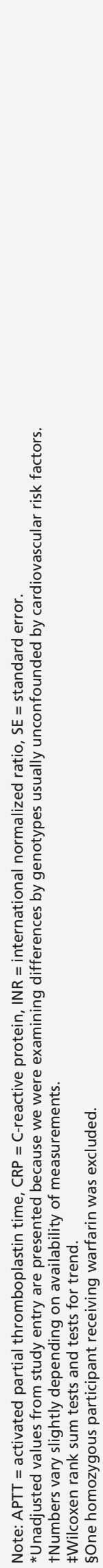 \\
\hline
\end{tabular}




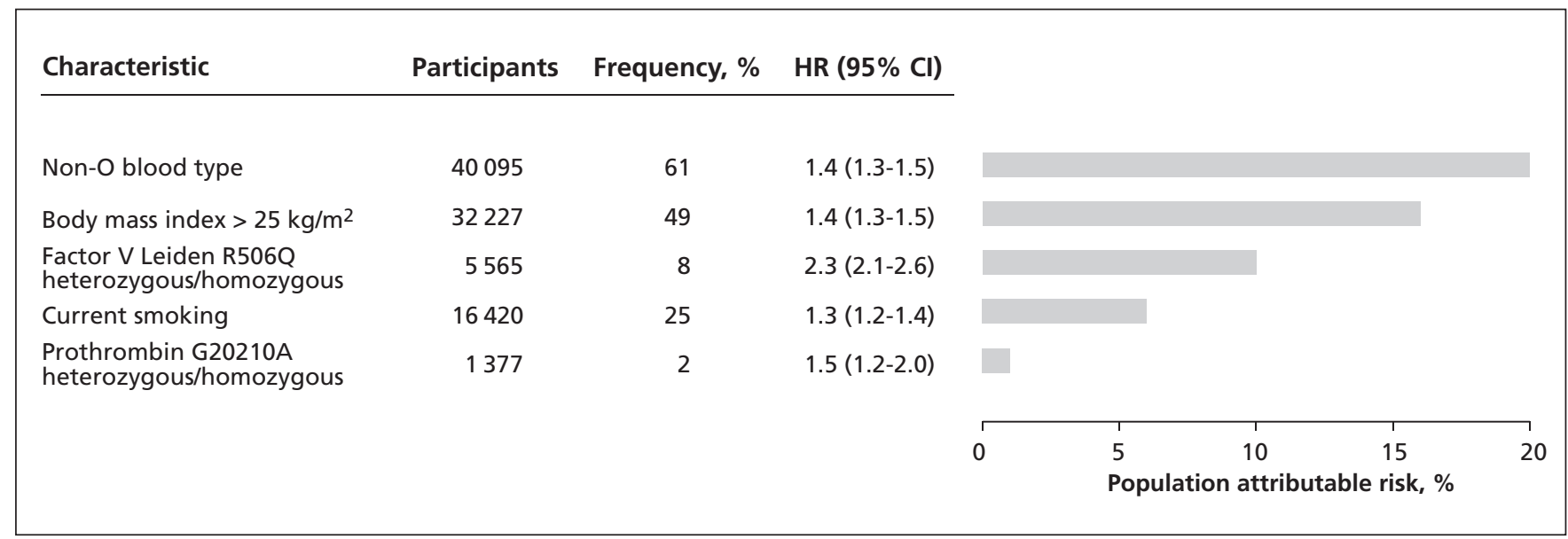

Figure 3: Ranking of risk factors for venous thromboembolism by population attributable risk in the general population. The results are based on 66001 participants in the Copenhagen General Population Study and the Copenhagen City Heart Study. All risk factors were dichotomized before analysis. Hazard ratios (HR) with $95 \%$ confidence intervals (Cls) were calculated using Cox regression models with age as the underlying time scale. Only significant HRs were included in the model; sex, hypertension and diabetes mellitus were considered but were not statistically significant.

Another potential limitation is the risk of misclassification of genotypes and diagnoses. Thus, some venous thromboembolism events may not have been recorded at hospitals, which is less likely for myocardial infarction. We determined ABO blood type by genotyping the single nucleotide polymorphisms specific to the 3 major alleles of ABO. Although these polymorphisms are mostly conserved, there are exceptions. For example, there are $\mathrm{O}$ alleles that lack the $261 \mathrm{delG}$ mutation analyzed in our study. Therefore, the frequency of the $\mathrm{O}$ allele may have been slightly underestimated. However, the frequencies of the $\mathrm{A}, \mathrm{B}, \mathrm{AB}$ and $\mathrm{O}$ alleles were consistent with those seen in the Danish general population. ${ }^{23}$ We confirmed the genotyping results by DNA sequencing, and all genotypes were in Hardy-Weinberg equilibrium. Diagnoses of venous thromboembolism and myocardial infarction have previously been validated. ${ }^{33,34}$ We therefore believe that misclassification of genotypes and diagnoses is a minor problem in our study.

Finally, because few individuals were homozygous for factor $\mathrm{V}$ Leiden and prothrombin G20210A mutations, interpretation of the results from these subgroups should be done with caution.

\section{Conclusion}

We found an additive effect of ABO blood type on risk of venous thromboembolism when combined with factor V Leiden R506Q and prothrombin G20210A; ABO blood type was the most important risk factor for venous thromboembolism in the general population. This suggests that $\mathrm{ABO}$ blood type should be considered for inclusion in genetic screening for thrombophilia.

\section{References}

1. Reilly MP, Li M, He J, et al. Identification of ADAMTS7 as a novel locus for coronary atherosclerosis and association of ABO with myocardial infarction in the presence of coronary atherosclerosis: two genome-wide association studies. Lancet 2011; 377:383-92.

2. Trégouët DA, Heath S, Saut N, et al. Common susceptibility alleles are unlikely to contribute as strongly as the FV and ABO loci to VTE risk: results from a GWAS approach. Blood 2009; 113:5298-303.

3. Dentali F, Sironi AP, Ageno W, et al. Non-O blood type is the commonest genetic risk factor for VTE: results from a metaanalysis of the literature. Semin Thromb Hemost 2012;38:535-48.

4. Jick H, Slone D, Westerholm B, et al. Venous thromboembolic disease and ABO blood type. A cooperative study. Lancet 1969; 1:539-42.

5. Medalie JH, Levene C, Papier C, et al. Blood groups, myocardial infarction and angina pectoris among 10,000 adult males. $N$ Engl J Med 1971;285:1348-53.

6. Talbot S, Wakley EJ, Langman MJ. A19 A29 B, and O bloodgroups, Lewis blood-groups, and serum triglyceride and cholesterol concentrations in patients with venous thromboembolic disease. Lancet 1972;1:1152-4.

7. Jenkins PV, O'Donnell JS. ABO blood group determines plasma von Willebrand factor levels: A biologic function after all? Transfusion 2006;46:1836-44.

8. O'Donnell J, Boulton FE, Manning RA, et al. Amount of H antigen expressed on circulating von Willebrand factor is modified by $\mathrm{ABO}$ blood group genotype and is a major determinant of plasma von Willebrand factor antigen levels. Arterioscler Thromb Vasc Biol 2002;22:335-41.

9. Orstavik KH, Magnus P, Reisner H, et al. Factor VIII and factor IX in a twin population. Evidence for a major effect of ABO locus on factor VIII level. Am J Hum Genet 1985;37:89-101.

10. Orstavik KH. Genetics of plasma concentration of von Willebrand factor. Folia Haematol Int Mag Klin Morphol Blutforsch 1990;117:527-31.

11. Shima M, Fujimura Y, Nishiyama T, et al. ABO blood group genotype and plasma von Willebrand factor in normal individuals. Vox Sang 1995;68:236-40.

12. Barbalic M, Dupuis J, Dehghan A, et al. Large-scale genomic studies reveal central role of ABO in sP-selectin and sICAM-1 levels. Hum Mol Genet 2010;19:1863-72.

13. Chung CM, Wang RY, Chen JW, et al. A genome-wide association study identifies new loci for ACE activity: potential implications for response to ACE inhibitor. Pharmacogenomics J 2010; 10:537-44.

14. Paterson AD, Lopes-Virella MF, Waggott D, et al. Genome-wide association identifies the $\mathrm{ABO}$ blood group as a major locus associated with serum levels of soluble E-selectin. Arterioscler Thromb Vasc Biol 2009;29:1958-67.

15. Qi L, Cornelis MC, Kraft P, et al. Genetic variants in ABO blood group region, plasma soluble E-selectin levels and risk of type 2 
diabetes. Hum Mol Genet 2010;19:1856-62.

16. Teupser D, Baber R, Ceglarek U, et al. Genetic regulation of serum phytosterol levels and risk of coronary artery disease. Circ Cardiovasc Genet 2010;3:331-9.

17. Ridker PM, Hennekens $\mathrm{CH}$, Lindpaintner $\mathrm{K}$, et al. Mutation in the gene coding for coagulation factor $\mathrm{V}$ and the risk of myocardial infarction, stroke, and venous thrombosis in apparently healthy men. N Engl J Med 1995;332:912-7.

18. Ridker PM, Hennekens CH, Miletich JP. G20210A mutation in prothrombin gene and risk of myocardial infarction, stroke, and venous thrombosis in a large cohort of US men. Circulation 1999;99:999-1004.

19. Ye Z, Liu EH, Higgins JP, et al. Seven haemostatic gene polymorphisms in coronary disease: meta-analysis of 66,155 cases and 91,307 controls. Lancet 2006;367:651-8.

20. Zacho J, Tybjaerg-Hansen A, Jensen JS, et al. Genetically elevated C-reactive protein and ischemic vascular disease. $N$ Engl J Med 2008;359:1897-908.

21. Nordestgaard BG, Benn M, Schnohr P, et al. Nonfasting triglycerides and risk of myocardial infarction, ischemic heart disease, and death in men and women. JAMA 2007;298:299-308.

22. Zacho J, Tybjaerg-Hansen A, Nordestgaard BG. C-reactive protein and risk of venous thromboembolism in the general population. Arterioscler Thromb Vasc Biol 2010;30:1672-8.

23. ABOblood.com. ABO Blood types reported racial and ethnic distribution. Available: www.aboblood.com (accessed 2012 Nov. 28).

24. Juul K, Tybjaerg-Hansen A, Schnohr P, et al. Factor V Leiden and the risk for venous thromboembolism in the adult Danish population. Ann Intern Med 2004;140:330-7.

25. Weischer M, Juul K, Zacho J, et al. Prothrombin and risk of venous thromboembolism, ischemic heart disease and ischemic cerebrovascular disease in the general population. Atherosclerosis 2010;208:480-3.

26. Ohira T, Cushman M, Tsai MY, et al. ABO blood group, othe risk factors and incidence of venous thromboembolism: the Longitudinal Investigation of Thromboembolism Etiology (LITE). $J$ Thromb Haemost 2007;5:1455-61.

27. Eichinger S, Hron G, Bialonczyk C, et al. Overweight, obesity, and the risk of recurrent venous thromboembolism. Arch Intern Med 2008;168:1678-83

28. Koster T, Blann AD, Briet E, et al. Role of clotting factor VIII in effect of von Willebrand factor on occurrence of deep-vein thrombosis. Lancet 1995:345:152-5.

29. Nossent AY, VAN Marion V, VAN Tilburg NH, et al. von Willebrand factor and its propeptide: the influence of secretion and clearance on protein levels and the risk of venous thrombosis. $J$ Thromb Haemost 2006;4:2556-62.
30. Oriol R, Mollicone R, Coullin P, et al. Genetic regulation of the expression of $\mathrm{ABH}$ and Lewis antigens in tissues. APMIS Suppl 1992;27:28-38

31. Wu O, Bayoumi N, Vickers MA, et al. $\mathrm{ABO}(\mathrm{H})$ blood groups and vascular disease: a systematic review and meta-analysis. $J$ Thromb Haemost 2008;6:62-9.

32. Franco RF, Trip MD, ten Cate $\mathrm{H}$, et al. The $20210 \mathrm{G}->$ A mutation in the 3 -untranslated region of the prothrombin gene and the risk for arterial thrombotic disease. Br J Haematol 1999;104:50-4

33. Juul K, Tybjaerg-Hansen A, Marklund S, et al. Genetically reduced antioxidative protection and increased ischemic heart disease risk: The Copenhagen City Heart Study. Circulation 2004;109:59-65.

34. Madsen M, Davidsen M, Rasmussen S, et al. The validity of the diagnosis of acute myocardial infarction in routine statistics: a comparison of mortality and hospital discharge data with the Danish MONICA registry. J Clin Epidemiol 2003;56:124-30.

Affiliations: From the Department of Clinical Biochemistry (Sode, Allin, Nordestgaard) and Anaesthesia (Sode), Herlev Hospital, Copenhagen University Hospital; the Department of Clinical Biochemistry (Dahl), Rigshospitalet, Copenhagen University Hospital; the Bispebjerg Hospital (Gyntelberg, Nordestgaard), Copenhagen University Hospital; and the Faculty of Health Sciences (Sode, Allin, Dahl, Gyntelberg, Nordestgaard), University of Copenhagen, Copenhagen, Denmark.

Contributors: Børge Nordestgaard initiated the study, which he designed along with Birgitte Sode and Morten Dahl. Data collection was performed by Finn Gyntelberg, Morten Dahl and Børge Nordestgaard. Laboratory and statistical analyses were performed by Birgitte Sode, Morten Dahl and Kristine Allin. All authors contributed to the interpretation of data. Birgitte Sode drafted the manuscript, which was revised and accepted by the other authors. All of the authors approved the final version submitted for publication.

Funding: The study was supported by the Danish Lung Association and Herlev Hospital, Copenhagen University Hospital. The study sponsors had no role in the design of the study, the collection, analysis or interpretation of data, the writing of the report or the decision to submit the article for publication. 